\title{
NIMROD: A computational laboratory for studying nonlinear fusion magnetohydrodynamics ${ }^{\text {a) }}$
}

\author{
C. R. Sovinec ${ }^{\text {b) }}$ \\ Engineering Physics Department, University of Wisconsin, Madison, Wisconsin 53706
}

T. A. Gianakon

Los Alamos National Laboratory, Los Alamos, New Mexico 87545

E. D. Held

Physics Department, Utah State University, Logan, Utah 84322

S. E. Kruger and D. D. Schnack

Science Applications International Corporation, San Diego, California 92121-1578

The NIMROD Team

(Received 11 November 2002; accepted 19 December 2002)

\begin{abstract}
Nonlinear numerical studies of macroscopic modes in a variety of magnetic fusion experiments are made possible by the flexible high-order accurate spatial representation and semi-implicit time advance in the NIMROD simulation code [A. H. Glasser et al., Plasma Phys. Controlled Fusion 41, A747 (1999)]. Simulation of a resistive magnetohydrodynamics mode in a shaped toroidal tokamak equilibrium demonstrates computation with disparate time scales, simulations of discharge 87009 in the DIII-D tokamak [J. L. Luxon et al., Plasma Physics and Controlled Nuclear Fusion Research 1986 (International Atomic Energy Agency, Vienna, 1987), Vol. I, p. 159] confirm an analytic scaling for the temporal evolution of an ideal mode subject to plasma- $\beta$ increasing beyond marginality, and a spherical torus simulation demonstrates nonlinear free-boundary capabilities. A comparison of numerical results on magnetic relaxation finds the $n=1$ mode and flux amplification in spheromaks to be very closely related to the $m=1$ dynamo modes and magnetic reversal in reversed-field pinch configurations. Advances in local and nonlocal closure relations developed for modeling kinetic effects in fluid simulation are also described. (C) 2003 American Institute of Physics. [DOI: 10.1063/1.1560920]
\end{abstract}

\section{INTRODUCTION}

Numerical simulation is an important tool for understanding the physics of magnetically confined plasmas, and it plays a particularly significant role in the area of magnetohydrodynamic (MHD) instabilities where wavelengths are comparable to the spatial scales of the device. These macroscopic modes are sensitive to the geometry of the equilibrium and its container in the linear regime, and they often lead to significant if not catastrophic changes to the magnetic configuration during their nonlinear evolution. While analytic theory lays a foundation for understanding the underlying physics, nonlinearity and geometry prevent general evaluation of theoretical models without the use of numerical solution techniques.

Realizing the full potential of simulation to provide solutions in device-specific configurations requires a sophisticated numerical representation of the spatial domain. This requirement and the ability to cope with physical effects at disparate time scales have guided development of the NIMROD code (Non-Ideal Magnetohydrodynamics with Rotation, Open Discussion). ${ }^{1,2}$ The implemented algorithm

\footnotetext{
a)Paper GI1 4, Bull. Am. Phys. Soc. 47, 135 (2002).

${ }^{b)}$ Invited speaker.
}

solves the nonlinear resistive MHD equations with extension to non-MHD effects accomplished through closure relations. Three-dimensional (3D) spatial approximation is achieved with 2D Lagrange-type finite elements in combination with finite Fourier series for the third dimension, which is assumed to be periodic and symmetric. This representation allows modeling of toroidal, cylindrical, and periodic linear configurations with arbitrarily shaped cross sections in each case. The code's use of finite-element basis functions of arbitrary polynomial degree permits high-order convergence for modeling the extreme anisotropies of nonideal magnetohydrodynamics. ${ }^{2}$ Furthermore, using nonuniform mesh spacing to represent complicated geometries and to meet resolution requirements efficiently does not diminish convergence rates. The semi-implicit temporal advance in NIMROD eliminates time-step restrictions associated with waves and (parallel) transport effects, which makes the code effective for solving the stiff partial differential equation systems that model high-temperature plasmas.

The geometric flexibility and multiple time-scale capabilities of the NIMROD code are meeting simulation needs for macroscopic behavior in tokamaks, spheromaks, and reversed-field pinches (RFP), and the code is beginning to find application outside of magnetic fusion energy research. The cumulative NIMROD effort is therefore analogous to an 


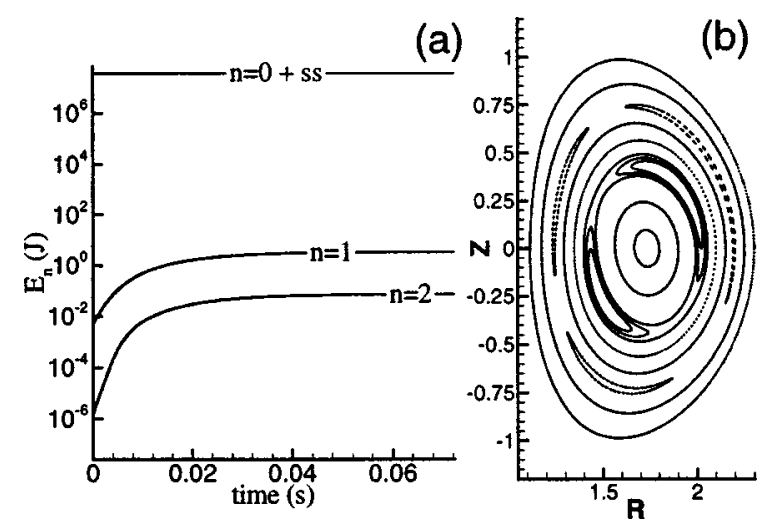

FIG. 1. Evolution of magnetic energy, separated by Fourier component, from the resistive-MHD tokamak simulation is shown in (a), where energies for the $n=0$ and steady-state fields have been added. The Poincare surface of section for the final magnetic field is shown in (b).

entire laboratory with a number of scientifically related activities. Here, we present a limited survey of fusion MHD studies conducted with NIMROD. [A recent investigation of DIII-D (Ref. 3) experimental discharges using NIMROD is reported in a separate article. ${ }^{4}$ ] In Sec. II, we describe simulation results for tokamaks and spherical tori. Modeling of magnetic relaxation in alternates is discussed in Sec. III. Our overall conclusions from the NIMROD effort appear in Sec. IV.

\section{TOKAMAK AND SPHERICAL TORUS SIMULATION}

\section{A. Resistive evolution of a magnetic island}

When magnetic islands form in tokamaks, they impact energy confinement, ${ }^{5}$ limit the achieved plasma- $\beta,{ }^{6,7}$ and the associated global magnetic perturbations interact with magnetic field errors, damping plasma rotation. ${ }^{8}$ Analyzing the evolution of magnetic islands and studying their consequences in realistic toroidal configurations requires numerical simulation, due to the geometry and nonlinear interactions.

An example NIMROD application to magnetic island evolution is a fixed-boundary simulation with a shaped equilibrium having a monotonic safety factor $(q)$ profile of 1.6 on axis and 7.8 at the wall that is unstable to a classical $(2,1)$ tearing mode. (The plasma pressure is artificially small to avoid stabilization. $\left.{ }^{9}\right)$ At dissipation parameters relevant to conventional tokamak startup operation $\left(S=\tau_{R} / \tau_{A}=10^{6}\right.$ and $\mathrm{Pm}=\tau_{v} / \tau_{R}=0.1$, where $\tau_{R}, \tau_{A}$, and $\tau_{v}$ are the resistive, Alfvénic, and viscous time scales, respectively), the calculated growth rate of the linear mode is $4.7 \times 10^{-4} \tau_{A}^{-1}$ for resistive $\mathrm{MHD}$, and nonlinear saturation occurs over a time scale that is nearly $1 / 10$ of the global resistive diffusion time [see Fig. 1(a)]. The evolution results in coupled island chains at the $q=2$ and $q=3$ surfaces, as shown in Fig. 1(b). While this realistic simulation requires parallel processing, it does not require an exceptionally large amount of computing resources. The spatial representation is a $32 \times 32$ mesh of radially packed biquartic finite elements with Fourier components $0 \leqslant n \leqslant 2$, and $2 \times 10^{4}$ time steps evolve the solution over a transport time scale. [The time step $(\Delta t)$ is restricted

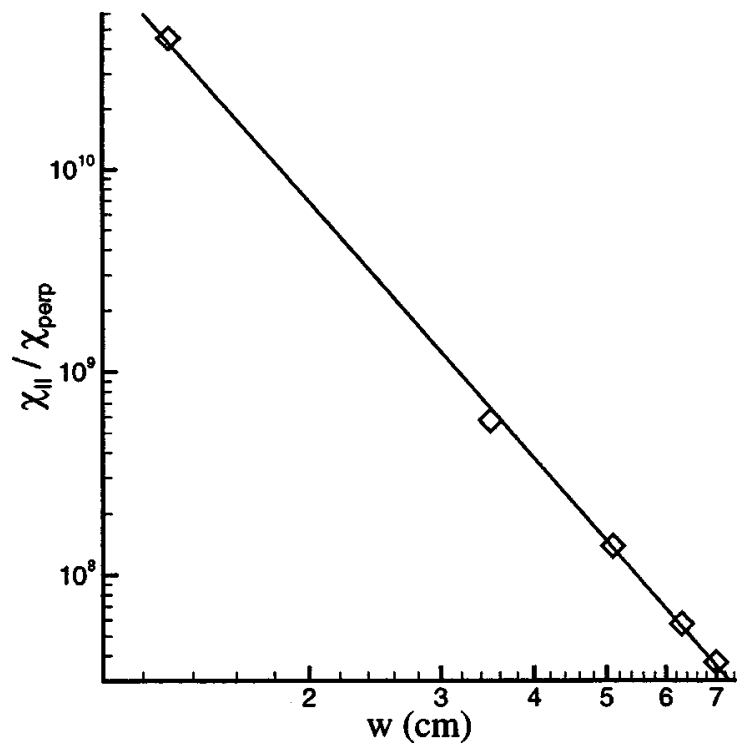

FIG. 2. Ratio of thermal diffusivities, $\chi_{\|} / \chi_{\perp}$, required to create an inflection of the temperature profile at the $q=2$ magnetic island chain vs island width measured on the outboard side at the toroidal angle where the $o$ point of the island crosses $Z=0$.

to accommodate present limitations in NIMROD's parallel matrix solves and not accuracy or numerical stability. In comparison, an explicit computation would require approximately $2 \times 10^{8}$ time steps for the resistive MHD part of the problem alone.]

Provided some form of linear or nonlinear excitation of a magnetic island, parallel thermal conduction within the island affects energy confinement, ${ }^{5}$ and anisotropy leads to an important threshold mechanism ${ }^{10}$ for neoclassical tearing modes (NTM). However, perpendicular conduction dominates parallel conduction within an island until the time scales for the two processes are comparable. This occurs when the island width is sufficiently large so that the parallel and perpendicular length scales $\left(L_{\|}\right.$and $\left.L_{\perp}\right)$ are related by $L_{\|} \sim L_{\perp} \sqrt{\chi_{\|} / \chi_{\perp}}$, where $\chi_{\|}$and $\chi_{\perp}$ are the respective thermal diffusivity coefficients. The resulting scaling of the $\chi_{\|} / \chi_{\perp}$-ratio required for flattening temperature within an island has been predicted analytically as $\chi_{\|} / \chi_{\perp} \sim w_{d}^{-4}$ for cylindrical geometry, where $w_{d}$ is the magnetic island width. ${ }^{10}$ To investigate this effect in toroidal geometry, the tearingmode evolution described previously is frozen at five different island widths, while thermal conduction is computed with a parallel diffusivity coefficient that increases with time. For each width, we note the parallel diffusivity required to create an inflection of the temperature profile within the island, and plot the result as the critical $\chi_{\|} / \chi_{\perp}$-ratio vs island width at the outboard midplane in Fig. 2. The power-law fit of the simulation data is $\chi_{\|} / \chi_{\perp} \sim w_{d}^{-4.2}$. Agreement of the exponent to $5 \%$ indicates the expected applicability of the analytic scaling to toroidal geometry, where a narrow layer of magnetic stochasticity exists near the island $x$-point.

\section{B. Velocity-moment closures for kinetic effects}

Over the time scale of the example resistive tearing evolution, drift and transport processes are important, so the 
MHD ordering is not an appropriate basis for a plasma model. ${ }^{11}$ Therefore, model improvement for long time-scale behavior is an important part of the NIMROD project. In some cases, the most important non-MHD effects can be incorporated into moment equations with modified but fluidlike, i.e., local, closure relations. For example, an important transport effect in the magnetic induction equation for a torus is the electron stress associated with poloidal flow, resulting in bootstrap current. The effect is sensitive to the formation of magnetic islands, due to their impact on the pressure profile, and there is positive feedback which leads to the NTM instability. ${ }^{12}$ Three-dimensional aspects require simulation to analyze experimental findings. However, available neoclassical fluid closures are derived with flux-surface-average relations for 1.5D transport calculations (Ref. 13, for example) and are not directly useful for macroscopic simulations, where local information is necessary. To address this, a combined theoretical/numerical effort by the NIMROD Team and collaborators has investigated various simplified closures for modeling neoclassical electron stresses as local fluid-like relations. The results of this study show that expressing neoclassical forces for the electrons and ions $(\alpha=e, i)$ as

$$
-\nabla \cdot \Pi_{\alpha}=-\rho_{\alpha} \mu_{\alpha}\left\langle B^{2}\right\rangle \frac{\mathbf{V}_{\alpha} \cdot \hat{\mathbf{e}}_{\theta}}{\left(\mathbf{B} \cdot \hat{\mathbf{e}}_{\theta}\right)^{2}} \hat{\mathbf{e}}_{\theta}
$$

where $\rho_{\alpha}, \mu_{\alpha}$, and $\mathbf{V}_{\alpha}$ are the species mass density, viscous damping frequency, and flow velocity, respectively, correctly reproduce the kinetic effects that are important for the magnetic induction equation. ${ }^{14}$ With the incorporation of these "heuristic" closures, NIMROD simulations of DIII-D discharge 86144 demonstrate both the NTM stability threshold associated with anisotropic thermal conduction and the stabilizing resistive curvature effect.

Although fluid-like closures for kinetic effects are expected to prove practical in modeling some macroscopic dynamics, they cannot reproduce the long-range effects of nearly collisionless particles. A suitable approach for numerical simulation is to calculate closures for velocity-moment equations from drift kinetic equations, allowing for a maximal ordering of collisional and free-streaming terms. We are pursuing this approach by expanding velocity-space perturbations to the local Maxwellian in terms of eigenfunctions of the Lorentz scattering operator. ${ }^{15}$ The heat flux and stresses at each point in space are determined by integrating the appropriate moments of the kinetic distortion along paths parallel to magnetic field lines, where convergence of the integrals is provided by collisions and phase mixing. The NIMROD implementation therefore determines magnetic field-line trajectories "on the fly" in order to calculate selfconsistent flux distributions for the evolution of macroscopic dynamics. The implementation for electron heat flux has been applied in RFP configurations (see Ref. 15) and is now being used in NTM simulations for tokamaks. Future efforts will address the kinetic electron momentum flux, allowing first-principles calculation of neoclassical effects in magnetic island evolution.

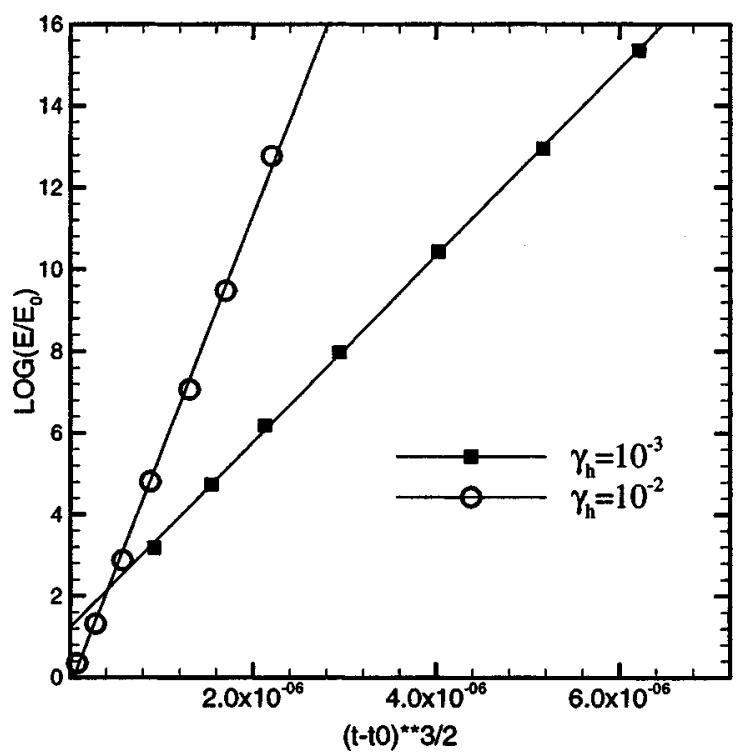

FIG. 3. Temporal dependence of magnetic fluctuation energy in tokamak simulations with heating effects. The two traces result from heating with $\gamma_{h}=0.01 \mathrm{~s}^{-1}$ and with $\gamma_{h}=0.001 \mathrm{~s}^{-1}$. Symbols show the simulation data, and solid lines are the best fits to $\exp \left[(t / \tau)^{3 / 2}\right]$.

\section{Disruption precursor simulation}

Modeling faster macroscopic phenomena in tokamak plasmas, including the onset of disruptive instability, is also of interest. We have used NIMROD to simulate the disruption precursor in DIII-D discharge 87009, which displayed faster-than-exponential growth during neutral beam heating. ${ }^{16}$ Analytic arguments based on a linear scaling of growth rate with plasma- $\beta$ for an ideal instability lead to an effective growth rate of $\gamma(t)=\hat{\gamma} \sqrt{\gamma_{h} t}$ with heating, where $\hat{\gamma}$ measures the dependence of the ideal mode's eigenvalue on $\beta,-\omega^{2}=\hat{\gamma}^{2}\left(\beta / \beta_{c}-1\right)$, and $\gamma_{h}$ is the heating rate. ${ }^{17}$ The resulting temporal dependence of the mode amplitude is proportional to $\exp \left[(t / \tau)^{3 / 2}\right]$ with the characteristic time, $\tau$, influenced by the heating rate, $\tau \equiv(3 / 2)^{2 / 3} \hat{\gamma}^{-2 / 3} \gamma_{h}^{-1 / 3}$. NIMROD simulations of this discharge (with the fitted equilibrium modified to make the ideal mode unstable with a fixed boundary at the separatrix) have been run with a heating source that is spatially proportional to the equilibrium pressure profile. NIMROD results for the heating rates $\gamma_{h}$ $=0.01 \mathrm{~s}^{-1}$ and $\gamma_{h}=0.001 \mathrm{~s}^{-1}$ are well fit by $\exp \left[(t / \tau)^{3 / 2}\right]$ behavior (see Fig. 3), and the resulting characteristic time, $\tau=(3 / 2)^{2 / 3} \hat{\gamma}^{-0.72} \gamma_{h}^{-0.28}$, compares favorably with the theoretical prediction.

\section{Modeling free-boundary effects}

By its nature, macroscopic activity tends to be sensitive to the location of the boundary. In this regard, the fixedboundary computations described above lose fidelity to the experiments they are intended to simulate, since the plasma is artificially constrained at the location of the separatrix of the symmetric equilibrium. Recent algorithmic development of numerically implicit temperature-dependent resistivity $\left(\sim T^{-3 / 2}\right)$ allows modeling the scrape-off-layer as cold resistive plasma without time-step limitation from the resulting 

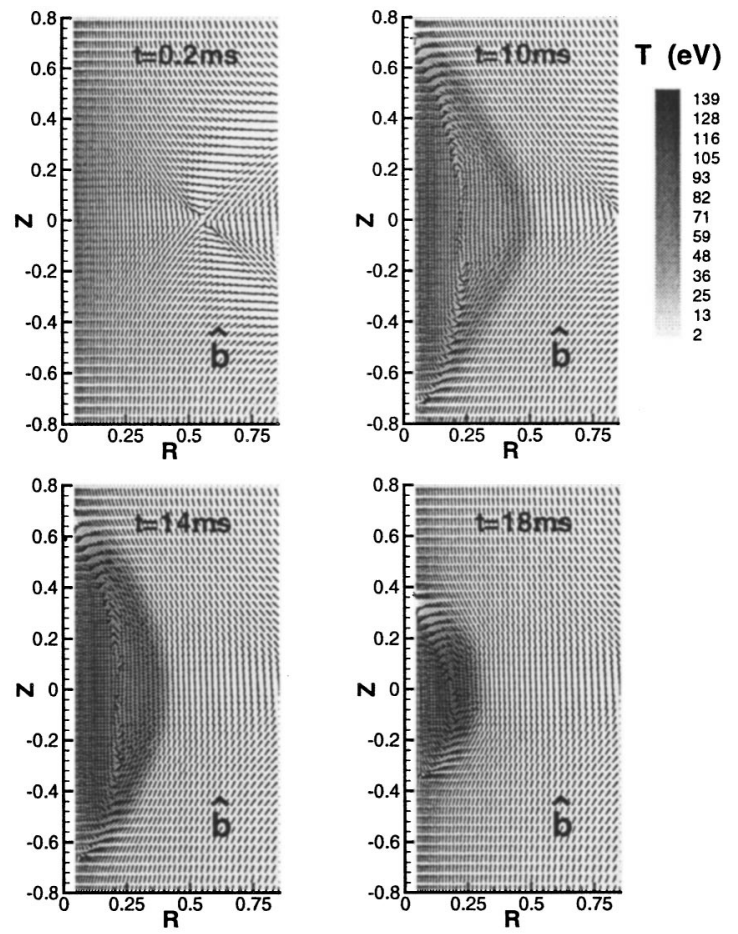

FIG. 4. Evolution of magnetic-field direction vectors and temperature in a 2D spherical torus computation with anisotropic thermal conduction and temperature-dependent resistivity.

large resistivity values on open magnetic field lines. This feature is being used to simulate the Pegasus spherical torus $^{18}$ at the University of Wisconsin, including the evolution of the equilibrium from Ohmic drive and the timedependent vertical field. The evolution of temperature and magnetic field from one simulation is shown in Fig. 4. Although most of the recent effort has been focused on making toroidally symmetric dynamics realistic for comparison with laboratory results from Pegasus, extension to $3 \mathrm{D}$ is straightforward with NIMROD and will be used to study the evolution of tearing modes with a self-consistent response in the equilibrium. This free-boundary capability is also improving our modeling of perturbed magnetic fields in tokamak simulations at conventional aspect ratios and permits simulating the dynamics of locking to field errors.

\section{MAGNETIC RELAXATION IN ALTERNATES}

\section{A. Spheromak relaxation}

The geometric flexibility of the finite element spatial representation makes NIMROD equally suitable for modeling spheromaks and RFPs, where the macroscopic activity has a fundamentally different role. Conventional operation in these devices drives axisymmetric equilibria beyond marginal stability for resistive MHD modes, and the ensuing macroscopic activity relaxes the current profile to create the configuration. For the spheromak, NIMROD simulations (without pressure evolution) were used in the first numerical study of sustainment from electrostatic current drive along open field lines ("helicity injection"). ${ }^{19,20}$ The results prove that nonlinear saturation of the $n=1$ mode, which is excited

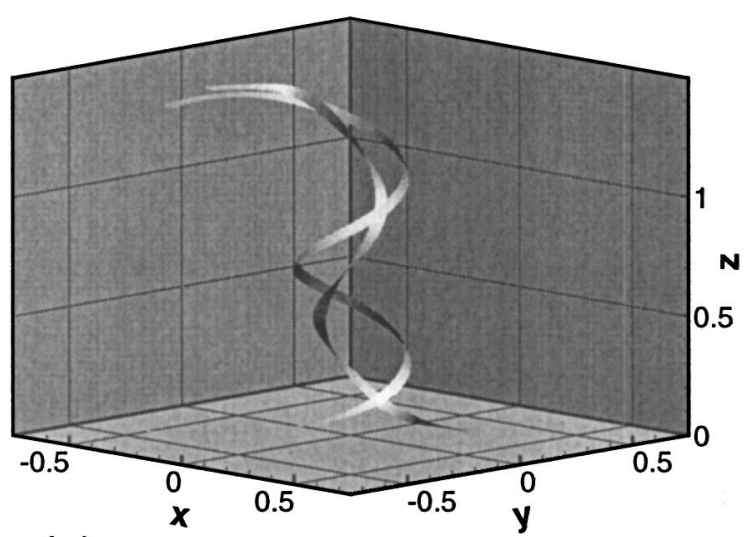

(a)

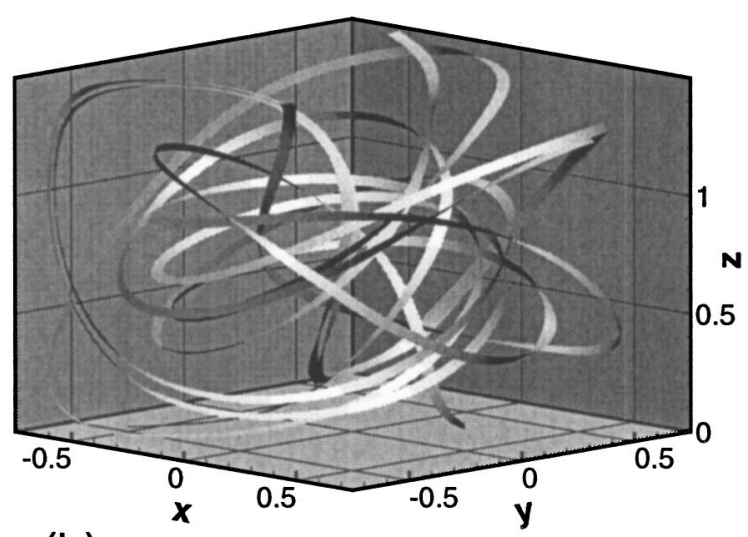

(b)

FIG. 5. Magnetic field-line traces from two points on the bottom (electrode) surface of an $S=5000$ pinch simulation for (a) the initial unstable axisymmetric configuration and (b) the saturated spheromak configuration.

by the pinch current on open magnetic field lines, converts toroidal magnetic flux into poloidal flux. This is accomplished through twisting and reconnection of magnetic field lines, as depicted by the magnetic field traces in Fig. 5. Comparing results for flux-core and magnetized Marshall-gun configurations shows that the flux conversion effect is qualitatively independent of electrode geometry, although quantitative differences occur. ${ }^{20}$ Final states with moderate drive or large dissipation parameters find steady sustainment of amplified flux with chaotic scattering of magnetic field lines throughout the volume. Increasing the current drive or decreasing dissipation leads to time-dependent relaxation oscillations, reminiscent of the increasingly intermittent behavior observed in RFP simulations as the $S$ value is increased. ${ }^{21}$

The $n=1$ mode has been observed in all strongly driven spheromak plasmas, ${ }^{22}$ but its role in the relaxation process was not fully appreciated before the NIMROD simulation study. A comparison between spheromak and RFP simulation results further clarifies the spheromak MHD. The appropriate spheromak/RFP comparison aligns the directly driven pinch current in each configuration by relating the $r-z$ plane of the spheromak with the $r-\phi$ plane of the RFP, as shown in Fig. 6. This comparison indicates that the toroidal direction of the spheromak is comparable to the azimuthal direction of a RFP, so the $n=1$ spheromak mode corresponds to the $m$ 

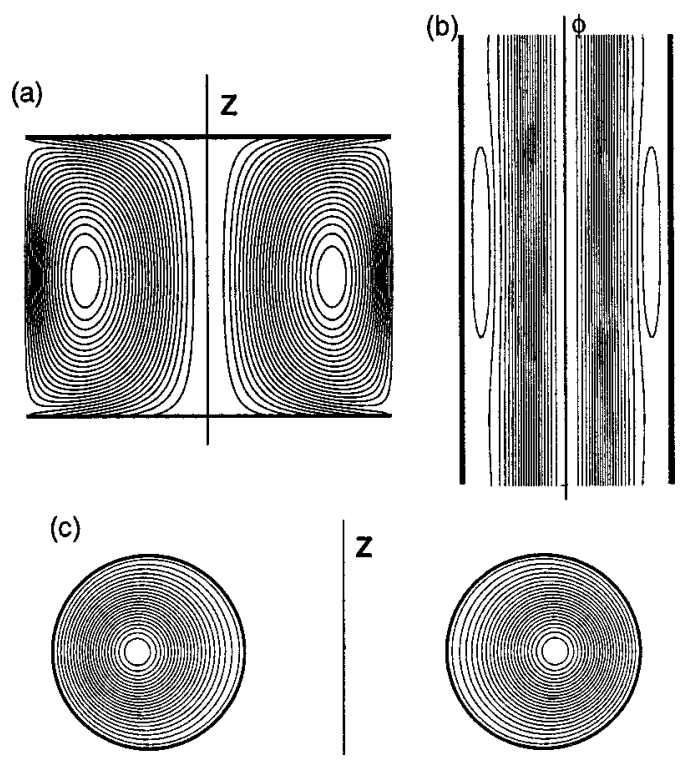

FIG. 6. NIMROD simulations of (a) average poloidal flux in a flux-core spheromak, (b) average toroidal flux in a cylindrical RFP (with periodic ends), and (c) average poloidal flux in a toroidal RFP. The applied electric field is in the vertical direction in (a) and (b), forming the basis for the spheromak-RFP analogy.

$=1$ dynamo modes of the RFP. Amplified poloidal flux in the spheromak is then a more extreme version of toroidal field reversal in a RFP. In fact, a RFP simulation with an unphysically small aspect ratio in a periodic cylinder and a flux-core spheromak simulation with the same dimensions, applied voltage, and net axial flux produce nearly equivalent amounts of flux conversion, despite the different boundary conditions at the ends of the cylinders. ${ }^{20}$ The comparison shows that line tying does not fundamentally alter the nonlinear MHD dynamics at spheromak parameters. Dominance of the $n=1$ mode during spheromak drive is a consequence of the dimensions of the container, the strong pinching of the current on open-field lines, and the global nature of the MHD mode. Scaling the $S$ value is then expected to decrease the length scales over which reconnection occurs, as indicated in Taylor's relaxation argument, ${ }^{23}$ without changing the dominant wave number in the system. With regard to the number of active modes, an aspect ratio scaling for the cylindrical RFP shows that the axial-wave-number fluctuation spectrum narrows as the cylinder length is decreased. ${ }^{24}$ The analogy then suggests that the spheromak will always have a narrower fluctuation spectrum than conventional RFPs.

The NIMROD spheromak simulations also show that removing the electrostatic drive leads to significant changes from the driven configuration. Without the electrostatic drive, the nonsymmetric perturbations decay more rapidly than the amplified symmetric poloidal flux, while the decay of poloidal flux induces toroidal current. The numerical results show that these effects lead to the formation of closed nested flux surfaces from states that have open magnetic field lines during sustainment. ${ }^{20}$ The implication is that confinement improves with decay while Ohmic heating continues. This is consistent with the fact that the highest temperatures in laboratory spheromaks have been measured during decay. ${ }^{22}$

\section{B. Toroidal effects in reversed-field pinches}

For the RFP itself, we have used NIMROD's flexible geometry to investigate toroidal effects by simulating the same plasma parameters in a circular cross-section torus as in a straight cylinder with periodic ends. Over a range of parameters, $\quad 1.6 \leqslant \Theta \leqslant 2$ and $1.1 \leqslant R / a \leqslant 3$, where $\Theta$ $\equiv B_{\theta}(a) /\left\langle B_{\phi}\right\rangle$ is the pinch parameter and $R$ for the cylinder is its length of periodicity divided by $2 \pi$, we find that standard multihelicity states are very similar in the two configurations. The simulations show that nonlinear coupling among resonant helicities, which is part of the normal RFP dynamo process, ${ }^{25}$ is stronger than coupling associated with toroidal geometry. While the MHD dynamics in multihelicity states are geometry independent, kinetic transport properties that are sensitive to variations in $|\mathbf{B}|$ along magnetic field-line trajectories are not. For standard RFP operation, toroidal geometry produces double-well structures along the ergodic path of magnetic field lines, due to $|\mathbf{B}|$ variations in both the poloidal and radial directions. ${ }^{15}$ The poloidal variations are sampled rapidly, while the radial variations associated with paramagnetism are sampled slowly with radial field-line diffusion.

When the dissipation parameters in cylindrical RFP simulations are sufficiently large, temporal oscillations damp, and laminar conditions are found. ${ }^{26}$ For similar parameters, NIMROD simulations reproduce laminar behavior in either cylindrical or toroidal geometry. However, while pure single-helicity states result from laminar conditions in the cylinder, pure single-helicity does not exist in a torus, due to the geometric coupling among different poloidal Fourier components ( $m$ numbers) for any toroidal Fourier component ( $n$ number). Simulations at $S=2000$ with varied viscosity $(\mathrm{Pm}=1,10$, and 100$)$, aspect ratio $(1.1 \leqslant R / a \leqslant 5)$, and pinch parameter $(1.4 \leqslant \Theta \leqslant 2)$ have been run in both configurations to find the impact of the toroidal couplinghere $\beta$ is set to zero for computational convenience. At Pm $=1(H=2000)$ and $\Theta \geqslant 1.6$, conditions are above the transition point to unsteady, multihelicity behavior. At $\mathrm{Pm}=10$, single-helicity states with nested helical flux surfaces result in cylindrical geometry, but the same conditions in toroidal geometry produce magnetic stochasticity over most of the domain, as shown in Figs. 7(a)-7(b). The symmetric part of the magnetic field exhibits reversal, i.e., $m=0$ perturbations are resonant, in both configurations at these parameters. In the cylinder, excitation of $m=0$ activity is suppressed by dissipation. However, an $m=0$ island chain is evident in the toroidal result, and the $n$ number of the chain matches that of the dominant, helical $m=1$ perturbation, so we infer that the excitation is geometric, rather than nonlinear. When $\mathrm{Pm}$ is increased to 100 or $\Theta$ is decreased to 1.4, the resulting mean field loses reversal, and nested helical flux surfaces exist in both configurations [Figs. 7(c)-(d)]. The correlation of reversal and stochastic magnetic field in toroidal geometry has been confirmed for $1.25 \leqslant R / a \leqslant 5$, so it is not limited to extremely small aspect ratio. 
(a)

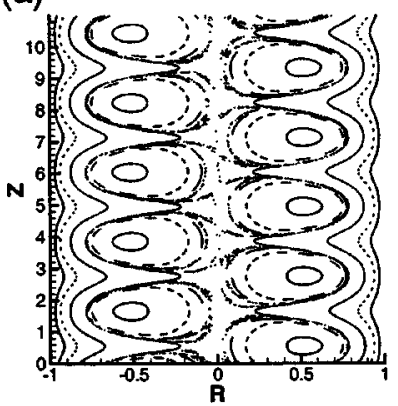

(c)

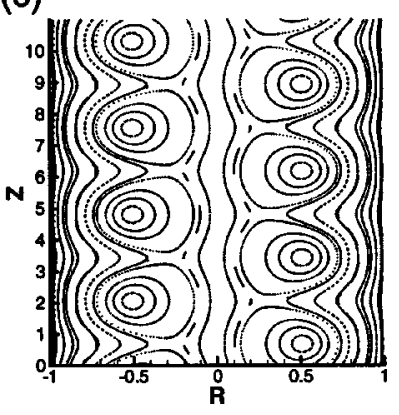

(b)

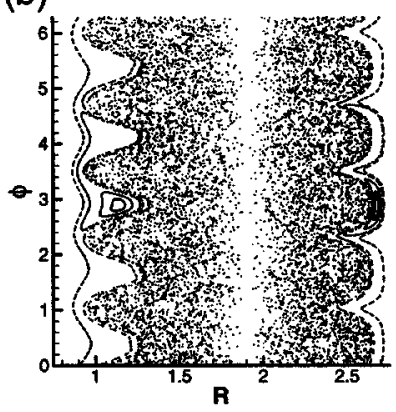

(d)

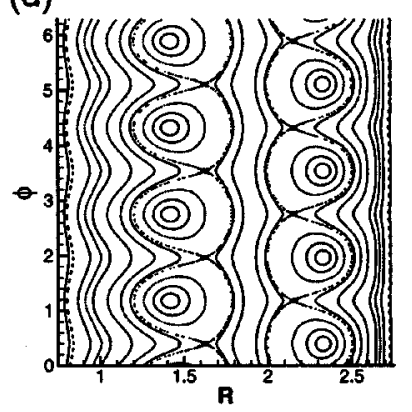

FIG. 7. Poincaré surfaces of section for magnetic field resulting in periodic pinch simulations with $S=2000, \mathrm{Pm}=10$, and $R / a=1.75$. Results are shown for (a) $\Theta=1.8$ and cylindrical geometry, (b) $\Theta=1.8$ and toroidal geometry, (c) $\Theta=1.4$ and cylindrical geometry, and (d) $\Theta=1.4$ and toroidal geometry.

\section{CONCLUSIONS}

The NIMROD applications described in this limited survey cover a range of magnetic and geometric configurations, and while all consider macroscopic modes in magnetic fusion plasmas, there is considerable variety in the research objectives for each study. This breadth is attributable to the flexible, high-order accurate spatial representation in NIMROD and the semi-implicit and implicit methods that allow solution of stiff systems in the extreme conditions of hightemperature plasmas. We have considered simulation studies that confirm the findings of analytic calculations in realistic configurations, like the anisotropic thermal conduction required to flatten temperature in magnetic islands, NTM stability thresholds, and faster-than-exponential growth of ideal modes. We have also considered studies that have brought out unanticipated results, like the dominant role and consequences of the $n=1$ mode in spheromak sustainment and toroidal geometry effects for laminar RFP conditions. Among other NIMROD applications are basic reconnection studies in sheared-slab geometry, dynamic current profile control in RFPs, modeling flows in a MHD thruster, and simulating AC helicity injection in a bowtie spheromak. We are also engaged in model development for incorporating ion gyrokinetic effects.

Each of the past and present numerical efforts can be characterized as taking advantage of NIMROD's simulation capabilities while driving concurrent development to provide better physics modeling and to address new applications. Simulations of resistive MHD evolution over drift and transport time scales, in particular, highlight conditions where

simple plasma descriptions are inadequate. The goal of simulating macroscopic activity in realistic conditions has then spurred development of the heuristic form of neoclassical stresses and closures based on self-consistent drift-kinetic calculations to provide kinetic effects in local fluid-moment evolution. For integrated plasma modeling, interaction of effects at multiple time scales is the central physics issue, and the NIMROD project will continue to emphasize its collaborative efforts that promote comprehensive nonlinear analysis.

\section{ACKNOWLEDGMENTS}

The authors wish to thank Curtis Bolton for initiating the NIMROD code development project and James Callen and Chris Hegna for many fruitful discussions regarding applications.

This work is supported by the U.S. Department of Energy.

${ }^{1}$ A. H. Glasser, C. R. Sovinec, R. A. Nebel, T. A. Gianakon, S. J. Plimpton, M. S. Chu, D. D. Schnack, and the NIMROD Team, Plasma Phys. Controlled Fusion 41, A747 (1999).

${ }^{2}$ C. R. Sovinec, A. H. Glasser, T. A. Gianakon, D. C. Barnes, R. A. Nebel, S. E. Kruger, D. D. Schnack, A. Tarditi, S. J. Plimpton, M. S. Chu, and the NIMROD Team, "Nonlinear magnetohydrodynamic simulation using high-order finite elements," J. Comput. Phys. (submitted).

${ }^{3}$ J. L. Luxon, R. Anderson, F. Batty et al., Plasma Physics and Controlled Nuclear Fusion Research 1986 (International Atomic Energy Agency, Vienna, 1987), Vol. I, p. 159.

${ }^{4}$ D. P. Brennan, R. J. La Haye, A. D. Turnbull, M. S. Chu, T. H. Jensen, L. L. Lao, T. C. Luce, P. A. Politzer, E. J. Strait, S. E. Kruger, and D. D. Schnack, Phys. Plasmas 10, 1643 (2003).

${ }^{5}$ Z. Chang and J. D. Callen, Nucl. Fusion 30, 219 (1990).

${ }^{6}$ Z. Chang, J. D. Callen, C. C. Hegna et al., Phys. Rev. Lett. 74, 4663 (1995).

${ }^{7}$ O. Sauter, R. J. LaHaye, Z. Chang et al., Phys. Plasmas 4, 1654 (1997).

${ }^{8}$ J. M. Finn and C. R. Sovinec, Phys. Plasmas 5, 461 (1998).

${ }^{9}$ A. H. Glasser, J. M. Greene, and J. L. Johnson, Phys. Fluids 19, 567 (1976).

${ }^{10}$ R. Fitzpatrick, Phys. Plasmas 2, 825 (1995).

${ }^{11}$ R. D. Hazeltine and J. D. Meiss, Plasma Confinement (Addison-Wesley, Redwood City, 1992), pp. 108-113.

${ }^{12}$ R. Carrera, R. D. Hazeltine, and M. Kotschenreuther, Phys. Fluids 29, 899 (1986); J. D. Callen, W. X. Qu, K. D. Siebert, B. A. Carreras, K. C. Shaing, and D. A. Spong, Plasma Physics and Controlled Nuclear Fusion Research 1986 (International Atomic Energy Agency, Vienna, 1987), Vol. II, p. 157.

${ }^{13}$ F. Hinton and R. Hazeltine, Rev. Mod. Phys. 48, 239 (1976)

${ }^{14}$ T. A. Gianakon, S. E. Kruger, and C. C. Hegna, Phys. Plasmas 9, 536 (2002).

${ }^{15}$ E. D. Held, J. D. Callen, C. C. Hegna, and C. R. Sovinec, Phys. Plasmas 8, 1171 (2001)

${ }^{16}$ M. S. Chu, J. M. Greene, L. L. Lao, R. L. Miller, A. Bondeson, O. Sauter, B. W. Rice, E. J. Strait, T. S. Taylor, and A. D. Turnbull, Phys. Rev. Lett. 77, 2710 (1996)

${ }^{17}$ J. D. Callen, C. C. Hegna, B. W. Rice, E. J. Strait, and A. D. Turnbull, Phys. Plasmas 6, 2963 (1999)

${ }^{18}$ R. Fonck, G. Garstka, T. Intrator, B. Lewicki, T. Thorson, R. Toonen, K. L. Tritz, B. White, and G. Winz, Bull. Am. Phys. Soc. 41, 1400 (1996).

${ }^{19}$ J. M. Finn, C. R. Sovinec, and D. Del-Castillo-Negrete, Phys. Rev. Lett. 85, 4538 (2000).

${ }^{20}$ C. R. Sovinec, J. M. Finn, and D. Del-Castillo-Negrete, Phys. Plasmas 8 , 475 (2001).

${ }^{21}$ S. Cappello and D. Biskamp, Nucl. Fusion 36, 571 (1996).

${ }^{22}$ T. R. Jarboe, Plasma Phys. Controlled Fusion 36, 945 (1994).

${ }^{23}$ J. B. Taylor, Phys. Rev. Lett. 33, 1139 (1974).

${ }^{24}$ Y. L. Ho, D. D. Schnack, P. Nordlund, S. Mazur, H.-E. Satherblom, J. Scheffel, and J. R. Drake, Phys. Plasmas 2, 3407 (1995).

${ }^{25}$ Y. L. Ho and G. G. Craddock, Phys. Fluids B 3, 721 (1991).

${ }^{26}$ S. Cappello and D. F. Escande, Phys. Rev. Lett. 85, 3838 (2000). 